Research Paper

\title{
Amelioration of Radiation-induced Skin Injury by HIV-TAT-Mediated Protein Transduction of RP-I from Rana pleurade
}

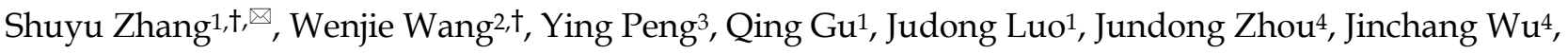 \\ Yinglong $\mathrm{Hou}^{5}$, Jianping $\mathrm{Cao}^{1}$ \\ 1. School of Radiation Medicine and Protection and Jiangsu Provincial Key Laboratory of Radiation Medicine and Protection, Medical \\ College of Soochow University, Suzhou 215123, China \\ 2. Cyrus Tang Hematology Center, Soochow University, Suzhou 215123, China \\ 3. Jiangsu Institute of Nuclear Medicine and Key Laboratory of Nuclear Medicine, Ministry of Health, Wuxi 214063, China \\ 4. Department of Radio-oncology, Nanjing Medical University Affiliated Suzhou Hospital, Suzhou 215001, China \\ 5. Department of Cardiology, Shandong Provincial Qianfoshan Hospital, Shandong University, Jinan, 250014, China \\ † The first two authors contributed equally to this work.
}

$\triangle$ Corresponding author: (S. Zhang), No. 199 Renai' Rd, Suzhou 215123, China. Tel/Fax: +86512-65880065, E-mail: zhang.shuyu@ hotmail.com

(c) Ivyspring International Publisher. This is an open-access article distributed under the terms of the Creative Commons License (http://creativecommons.org/ licenses/by-nc-nd/3.0/). Reproduction is permitted for personal, noncommercial use, provided that the article is in whole, unmodified, and properly cited.

Received: 2013.08.21; Accepted: 2013.12.11; Published: 2013.12.19

\begin{abstract}
Radiation-induced reactive oxygen species (ROS) can damage DNA and most other biological macromolecules in skin and radiation-induced skin injury is a serious concern for radiation therapy. Skin possesses an extremely efficient antioxidant system, which is conferred by two systems: antioxidant enzymes and small molecules that can scavenge ROS by donating electrons. Amphibian skin is a multifunctional organ, which protects against dangers of various oxidative stresses. Recently, a small peptide called RP-I was isolated from the skin secretions of Rana pleurade, which shows strong antioxidant activity. However, this RP-I peptide is limited because its inability to across the cell membrane. Protein transduction domains (PTDs) have demonstrated high efficiency for facilitating the internalization of both homologous and heterogeneous proteins into cells. This study aims to elucidate the protective effects of a HIV-TAT (TAT) PTD-coupled RP-I fusion protein (TAT-RPI) on radiation-induced skin injury in vitro and in vivo. The synthesized fusion TAT-RPI peptide can be incorporated into human keratinocyte $\mathrm{HaCaT}$ cells in a dose- and time-dependent manner without cytotoxicity. We then evaluated the protective role of TAT-RPI against ionizing radiation. TAT-RPI supplementation increased anti-superoxide anion ability of $\mathrm{HaCaT}$ cells and decreased $\mathrm{HaCaT}$ cell radiosensitivity to irradiation. Moreover, TAT-RPI was able to penetrate the skin of rats, entering epidermis as well as the dermis of the subcutaneous layer in skin tissue. Topical spread of TAT-RPI promoted the amelioration of radiation-induced skin damage in rats. These results suggest that TAT-RPI has potential as a protein therapy for radiation-induced skin injury.
\end{abstract}

Key words: protein transduction domain (PTD), HIV-TAT, RP-1, radiation-induced skin injury, $\mathrm{HaCaT}$ cells

\section{Introduction}

Radiotherapy has become a valuable alternative to primary surgical approaches for cancer patients
$(1,2)$. However, because skin is usually the first site of external radiation particles entry during radiotherapy, 
variable degrees of skin reactions can occur (3). The skin may be significantly injured during this process and its function profoundly impaired due to radiation-induced oxidative stress that damage DNA and most other biological macromolecules in skin $(10,11)$. Serious radiation-induced skin injuries can cause severe pain, secondary infection, ulceration, and even necrosis when intolerable doses are administered $(3,6)$. Therefore, radiation-induced skin injury remains a serious concern, which may limit the duration and dose of radiation treatment.

Oxidative stresses from extrinsic and intrinsic mechanisms cause skin damage $(7,8)$. Skin possesses an extremely efficient antioxidant system. This antioxidant activity consists of two systems: antioxidant enzymes and small molecules that can scavenge reactive oxygen species (ROS) by donating electrons $(9,10)$. For amphibians, the life transition is characterized by the presence of higher oxygen concentration, indicating more endogenous production of ROS $(11,12)$. However, amphibians have involved a multifunctional skin, which protects against dangers of oxidative stresses such as ultraviolet ray (11). Recently, a series of small peptide was isolated from the skin secretions of Rana pleurade, which show multifunctional properties, such as combined antioxidant, anti-inflammatory and antimicrobial activities $(13,14)$. These peptides are coded by their respective genes and different from antioxidant enzymes and low molecular weight antioxidants. Among these small peptides, RP-1 (AMRLTYNKPCLYGT) showed strong free radical scavenging ability (13), indicating a desire molecule for the treatment of ROS-induced skin damage.

However, most of proteins cannot be directly supplemented into cells due to the cytomembrane barrier. Protein transduction domains (PTDs) are a group of short peptides that possess the ability to efficiently penetrate cellular membranes $(14,15)$. Their transmembrane ability is attributed to their enrichment in arginine and lysine residues, which exhibits a characteristic positive charge. PTDs are able to facilitate the internalization of both homologous and heterogeneous cargo into cells and tissues $(16,17)$. HIV-TAT (TAT) domain (YGRKKRRQRRR) was identified in 1988 as the first PTD $(17,18)$. Subsequently, a variety of of PTDs have been reported, including VP22 protein from Herpes simplex virus. antennapedia homeoprotein from Drosophila and chemically synthesized 8-mers of polyarginine and polylysine $(19,20)$. The appearance of PTDs may have therapeutic implication in disease treatment.

Whether PTD-mediated delivery of the RP- 1 is able to effectively protect skin cells from ionizing radiation via scavenging intracellular ROS is unclear. In the present study, the synthesized TAT-RP1 fusion peptide was efficiently incorporated into human HaCaT cells in a dose- and time-dependent manner. We found that TAT-RP1 decreased radiosensitivity of $\mathrm{HaCaT}$ cells to irradiation. Moreover, TAT-RP1 was able to penetrate the skin of rats. Topical spread of TAT-RP1 ameliorated radiation-induced skin damage in rats. These results suggest that TAT-RP1 has potential as a protein therapy for radiation-induced skin injury.

\section{Materials and methods}

\section{Animals and treatments}

Male SD rats (4 weeks old) were purchased from Shanghai SLAC Laboratory Animal Co., Ltd. (Shanghai, China). Rats were anesthetized with an intraperitoneal injection of ketamine $(75 \mathrm{mg} / \mathrm{kg})$ and xylazine $(10 \mathrm{mg} / \mathrm{kg})$, and the hair on rat buttock skin was shaved using a razor. Rats were immobilized with adhesive tape on a plastic plate to minimize motion during irradiation exposure. A $3 \mathrm{~cm}$ thick piece of lead was used to shield the rats and localize the radiation field $(3 \mathrm{~cm} \times 4 \mathrm{~cm})$. A single dose of $45 \mathrm{~Gy}$ was administered to the treatment area of each rat at a dose rate of $750 \mathrm{cGy} / \mathrm{min}$ using a $6-\mathrm{MeV}$ electron beam accelerator (Clinac 2100EX; Varian Medical Systems Inc, CA). This dose was selected because it can significantly induce skin injury $(100 \%$ of animals develop grade 4-5 injury at this dose) (21). After irradiation, rats were randomly divided into three groups (5 animals per group): 1 ) rats were mock treated with PBS; 2) with a topical spread of $50 \mu \mathrm{g}$ RP-1 twice a week; 3) with a topical spread of $50 \mu \mathrm{g}$ TAT-RP1 twice a week. Skin reactions were followed at regular intervals using the semi-quantitative skin injury scale from 1 (no damage) to 5 (severe damage), as previously described (22). All the protocols and procedures were approved by the Animal Experimentation Ethics Committee of the Soochow University.

\section{Synthesis of peptides}

All of the peptides used in this work were synthesized by Shanghai Mocellbio Scientific Inc. (Shanghai, China) with a confirmed purity higher than $95 \%$ by HPLC and MALDI-TOF mass spectrometry.

\section{Transduction of TAT-RP I into cultured skin cells in vitro}

To determine whether the TAT-RP1 was internalized into cells, human keratinocyte HaCaT cells were used. The cells were maintained in Dulbecco's modified Eagle's medium (DMEM) supplemented with $10 \%$ FBS and antibiotics (100 units/ml penicillin $\mathrm{G}$ and 100 units/ml streptomycin sulfate; Gibco, 
Grand Island, NY). To analyze the transduction efficiency of the fusion protein, $\mathrm{HaCaT}$ cells were grown to $50 \%-60 \%$ confluence in a 6 -well plate. Thereafter, these cells were treated with PBS or RP-1 or TAT-RP1 labled by FITC. After the addition of peptide in the medium, the cells were incubated for different time points and then washed three times with PBS to remove free peptide. Images were collected with a Leica TCS SP2 confocal microscope (Leica, Germany).

\section{Cytotoxicity assay}

Cells $\left(2 \times 10^{3}\right)$ were seeded into 96-well plates in $0.1 \mathrm{ml}$ of DMEM supplemented with FBS and were incubated for $24 \mathrm{~h}$. The cells were then treated with indicated concentrations of TAT-RP1 and incubated for an additional $24 \mathrm{~h}$. Then the cells were incubated for $4 \mathrm{~h}$ with $200 \mu \mathrm{g} / \mathrm{ml} \mathrm{3-(4,5-dimethylthiazol-2-yl)-}$ 2,5-diphenyl-2H-tetrazolium bromide (MTT Sigma, St Louis, MO). The reagent was dissolved in dimethylsulfoxide (DMSO, Solon, OH). The absorbance was measured at $490 \mathrm{~nm}$ using a 96-well plate reader. All of the experiments were performed in triplicate.

\section{Anti-superoxide anion ability assay}

Cells were lysed by passive lysis buffer (Promega, Madison, WI). Anti-superoxide anion ability assay was performed according to the anti-superoxide anion ability assay kit (Nanjing Jiancheng, Nanjing, China).

\section{Clonogenic assay}

For standard clonogenic assays, cells were seeded into 6-well plates at 500-1,000 cells/well depending on the dose of radiation. Twenty-four hours after seeding, the cells were mock treated or treated with RP-1 or TAT-RP1 for $4 \mathrm{~h}$. The cells were then irradiated using a 6-MV X-ray linear accelerator (Clinac 2100EX; Varian Medical Systems Inc., CA) at a dose rate of $2 \mathrm{~Gy} / \mathrm{min}$; a $1.5-\mathrm{cm}$ bolus was used as a compensator. After irradiation, the peptide-containing medium was immediately replaced with fresh DMEM medium. The cells were then grown for 7-10 days to allow for colony formation, fixed and stained using crystal violet. Colonies consisting of 50 or more cells were counted as a clone.

\section{Statistical analysis}

Data were expressed as the mean \pm standard error of the mean (SEM) of at least three independent experiments. Standard error bars are included for all data points. The data were then analyzed using Student's $t$ test when only two groups were present, or assessed by one-way analysis of variance (ANOVA) when more than two groups were compared. The radiation sensitivity enhancement ratio (SER) was measured according to the multi-target single hit model. For ordinal data, the Mann-Whitney test was used. Statistical analysis was performed using SPSS software. Data were considered significant if $P<0.05$. Statistical analysis was performed using SPSS software (Release 17.0, SPSS Inc.).

\section{Results}

\section{Transduction of peptides into cultured $\mathrm{HaCaT}$ cells}

The primary structure of TAT, RP-1 and TAT-RP1 peptides are summarized in Fig.1A. To determine whether these peptides could be internalized into human HaCaT cells, cells were mock-treated or treated with FITC-labled TAT, RP-1 or TAT-RP1, respectively. Four hours after administration, the cells were washed three times with PBS to remove free peptides and those attached on cell surface. Under a fluorescent microscope, green fluorescence could be observed from cells treated with TAT-RP1 but not in the mock-treated HaCaT cells (Fig. 1B). Cells treated with RP-1 showed weak fluorescence in the cytoplasm (Fig. 1B and 1C, white arrow), indicating unavailability of the RP-1 peptide. However, when coupled with TAT, the fusion peptide was distributed in both nucleus and the cytoplasm of HaCaT cells (Fig. 1B and 1C), indicating that the TAT domain can be used as a delivery vehicle for RP-1 transfer.

\section{Dose- and time-dependent transduction of TAT-RP I}

To test the effectiveness of TAT-RP1 transduction, $\mathrm{HaCaT}$ cells were treated with $10 \mu \mathrm{M}$ TAT-RP1 at various time points. The protein began to enter the cell after incubation for $20 \mathrm{~min}$ and reached maximal uptake within $1 \mathrm{~h}$ (Fig. 2A and 2B). In addition, cells were treated with TAT-RP1 at different concentrations for $1 \mathrm{~h}$. The percentage of transduced TAT-RP1 was increased with the doses used to treat $\mathrm{HaCaT}$ cells (Fig. 2C and 2D). After $10 \mu \mathrm{M}$ TAT-RP1 treatment for $1 \mathrm{~h}$, nearly $100 \%$ of the HaCaT cells were incorporated with this exogenous peptide. These results suggest that TAT-RP1 could be quickly and efficiently transduced into cells in a dose- and time-dependent manner.

\section{The cytotoxicity of TAT-RPI on human HaCaT cells}

To evaluate the cytotoxic effects of RP-1 and TAT-RP1 on cultured human skin cells, we treated the HaCaT cells with different concentrations of RP-1 or TAT-RP1 and measured cell viability using an MTT assay. As shown in Fig. 2A, $10 \mu \mathrm{M}$ RP-1 showed weak cytotoxicity to $\mathrm{HaCaT}$ cells after $24 \mathrm{~h}$ incubation. Comparatively, 5 and $10 \mu \mathrm{M}$ TAT-RP1 did not induce significant cytotoxicity in $\mathrm{HaCaT}$ cells. 


\section{The effect of TAT-RP I on anti-superoxide anion ability of $\mathrm{HaCaT}$ cells}

To evaluate the anti-superoxide anion capability of TAT-RP1 on cultured human skin cells, we treated the HaCaT cells with RP-1 or TAT-RP1 for $4 \mathrm{~h}$ and measured total anti-superoxide anion ability of cells. Results revealed that compared with PBS-treated cells, TAT-RP1-treated cells showed $\sim 1.6$-fold significant increased anti-superoxide anion ability, while RP-1-treated cells did not exhibit such change (Fig. 3B). This result indicated that TAT-mediated internalization of RP-1 enhances antioxidant capability against superoxide anion radicals.

\section{The effect of TAT-RPI on the radiosensitivity of $\mathrm{HaCaT}$ cells}

To investigate the effect of TAT-RP1 on the radiosensitivity of $\mathrm{HaCaT}$ cells, we performed an in vitro clonogenic cell survival assay using TAT-RP1 treatment plus radiation. $\mathrm{HaCaT}$ cells pretreated with 10 $\mu \mathrm{M}$ TAT-RP1 plus X-ray irradiation exhibited significantly higher clonogenic survival rates than cells treated with radiation alone. Compared with mock-treated cells, the SER was 0.84 for cells treated with $10 \mu \mathrm{M}$ TAT-RP1 (Fig. 3C). In contrast, cells pretreated with RP-1 showed similar survival rates with cells treated with radiation alone.The data were further analyzed using two-way ANOVA to test the interaction effect between TAT-RP1 and radiation. Our results indicated that the interaction effect between 10 $\mu$ M TAT-RP1 treatment and radiation was statistically significant $(P<0.05)$ for HaCaT cells, suggesting that TAT-RP1 pretreatment could protect cells from X-ray irradiation. Taken together, these results demonstrate that pretreatment with TAT-RP1 could attenuate the radiosensitivity of human $\mathrm{HaCaT}$ cells.
A

RP-1 AMRLTYNKPCLYGT

TAT YGRKKRRQRRR

TAT-RP1 YGRKKRRQRRRAMRLTYNKPCLYGT

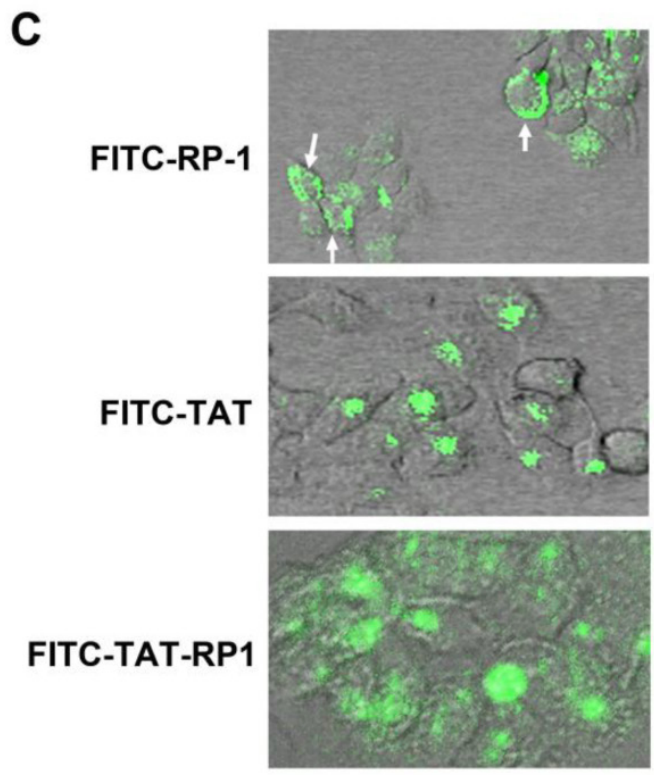

B
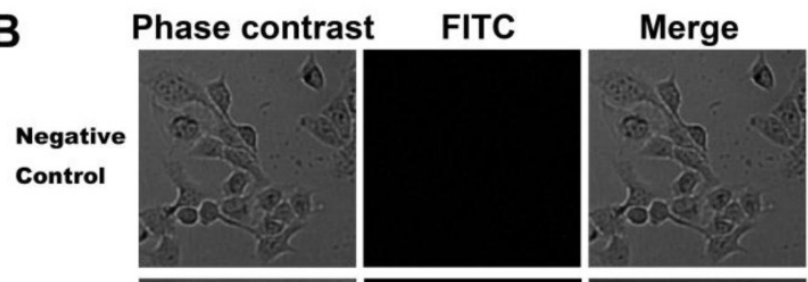

FITC-

Control
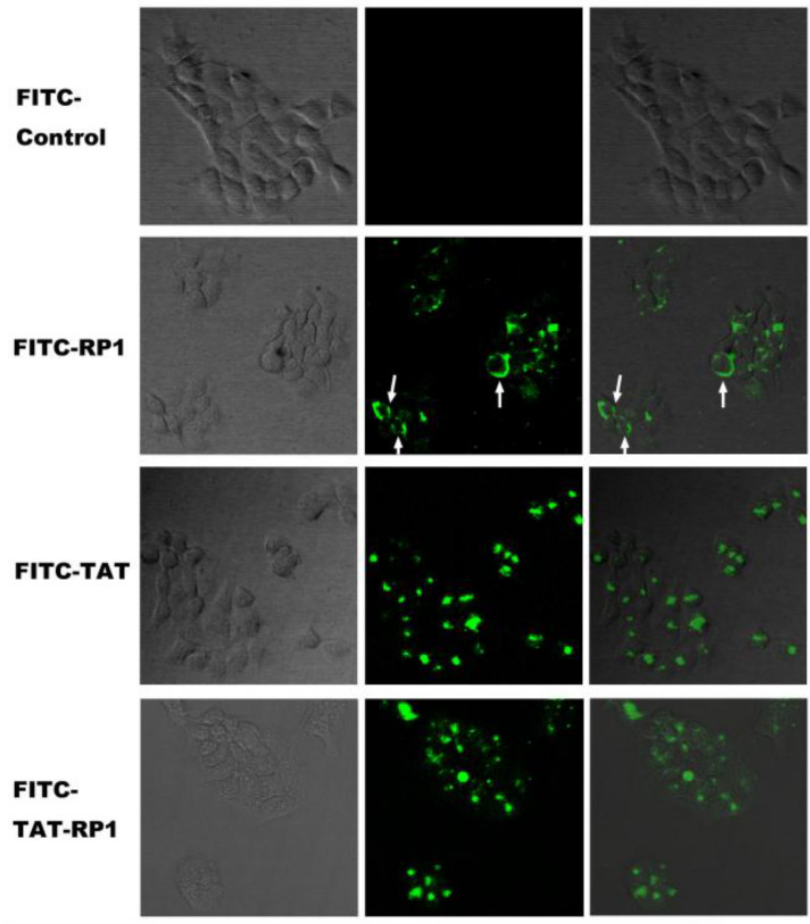

Figure I. Transduction of peptides into cultured HaCaT cells. (A) The primary structure of peptides in this study. (B) HaCaT cells were grown to confluence on a 24-well plate. Thereafter, these cells were mock-treated or treated with $10 \mu \mathrm{M}$ indicated peptide. Four hours after the addition of peptide into culture medium, cells were washed three times with PBS to remove free peptide. The distribution of fluorescence within cells was analyzed on a Leica confocal microscope. Fluorescent signals were obtained under the same conditions. (C) Enlarged image of cells treated with indicated peptides. 

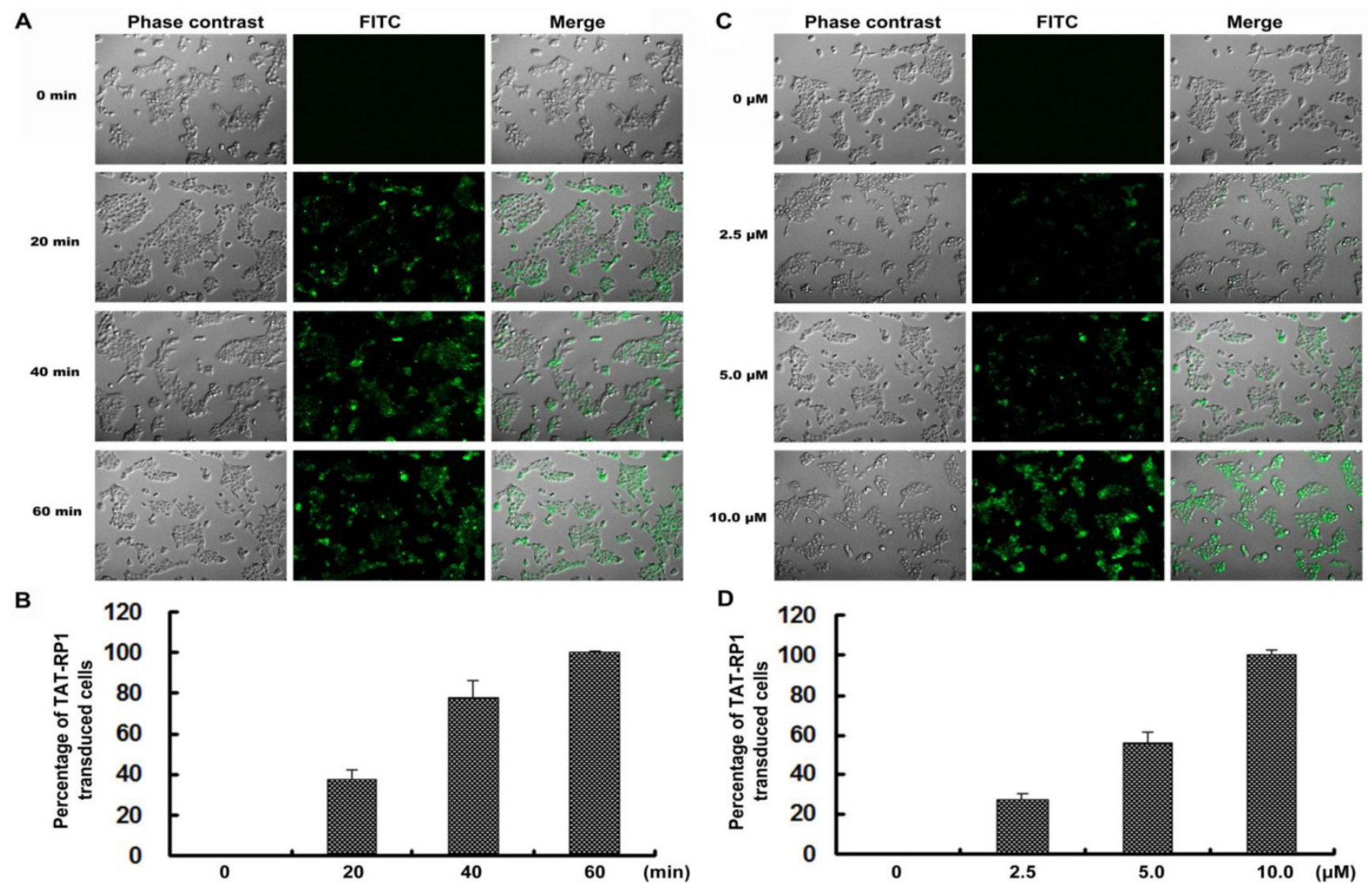

Figure 2. Time- and dose-dependent transduction of TAT-RPI into HaCaT cells. (A) HaCaT cells were treated with I0 $\mu$ M TAT-RPI for 0 to $60 \mathrm{~min}$. After the addition of TAT-RPI, cells were washed three times with PBS to remove free peptide. Fluorescent signals were obtained under the same conditions. (B) Percentage of TAT-RPI transduced cells. The data are shown as the mean values \pm SEM for three independent experiments. (C) HaCaT cells were treated with TAT-RPI at different concentrations for $60 \mathrm{~min}$. (D) Percentage of TAT-RPI transduced cells. The data are shown as the mean values \pm SEM for three independent experiments.
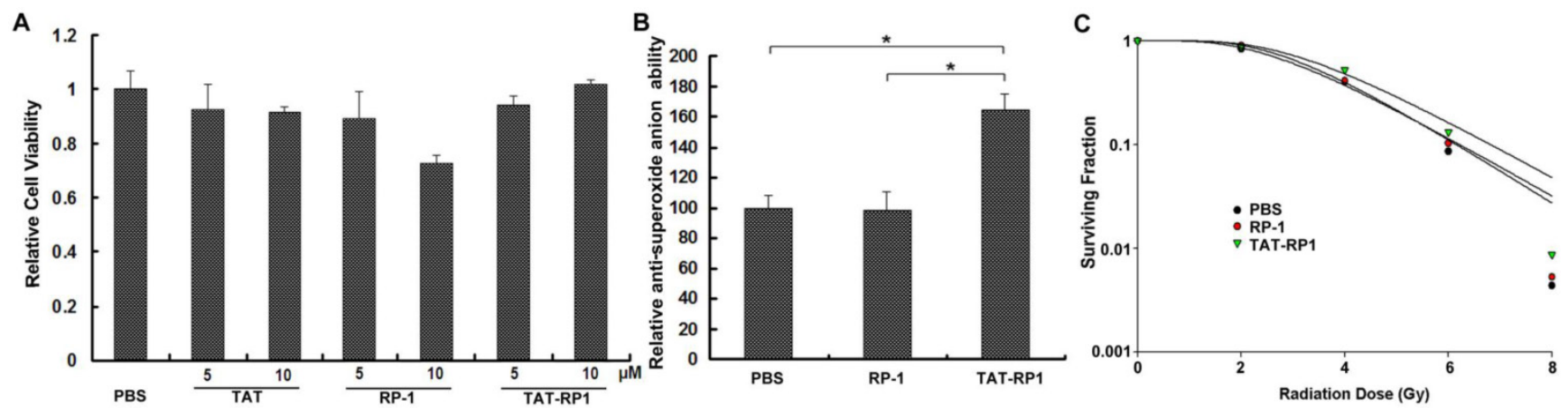

Figure 3. The effect of TAT-RPI on cytotoxicity, ROS scavenging and radiosensitivity of HaCaT cells. (A) Cytotoxicity of TAT-RPI on $\mathrm{HaCaT}$ cells. $\mathrm{HaCaT}$ cells were exposed to TAT-RPI for different time points. Cell survival was assessed using an MTT assay. The data are shown as the mean values \pm SEM for three independent experiments. (B) Anti-superoxide anion ability of HaCaT cells after treatment of PBS, $10 \mu M$ RP-I or $10 \mu M$ TAT-RPI for $4 \mathrm{~h}$. Anti-superoxide anion ability of cells was measured as described in the Material and Methods. (C) HaCaT cells were treated with the indicated concentrations of TAT-RPI for $4 \mathrm{~h}$ and then were exposed to 2, 4, 6 or $8 \mathrm{~Gy}$ irradiation. The survival data were normalized to that of the unirradiated control group. The radiation sensitivity enhancement ratio (SER) was calculated for HaCaT cells that were treated with $10 \mu M$ RP-I or TAT-RPI prior to X-ray irradiation.

\section{TAT-RP I ameliorated radiation-induced skin injury in vivo}

We then evaluated the ability of the TAT-RP1 fusion peptide to transduce into rat skin. The peptides were sprayed on rat skin and the degree of skin penetration of these peptides were analyzed a fluorescent microscopy. As shown in Fig. 4, the fluorescence signal in skin treated with TAT-RP1 fusion peptide was significantly detected in the epidermis as well as the dermis of the subcutaneous layer in skin tissue (blue arrow). This result demonstrates that the TAT-RP1 fusion peptide with HIV-TAT domain residues can 
not only be transduced into cultured skin cells but also penetrate the rat skin.

To determine whether TAT-RP1 could attenuate radiation-induced skin injury, we performed an experiment using a single dose of 45 Gy delivered to the buttock skin of SD rats as previously reported (21). Then, the irradiation field was topically spread by PBS, RP-1 or TAT-RP1 peptide. Injuries of skin tissues were graded on a scale of 1 (no damage) to 5 (severe damage) as reported $(21,22)$. Cutaneous damage was observed 4-5 days after irradiation and reached a maximum 10-14 days later. Radiation-induced skin injury was significantly less severe in the TAT-RP1-treated group, compared to the PBS-treated rats since 15 days post irradiation, while the RP-1-administrated rats showed similar skin tissue damage with that of the control group (Fig. 5A and 5B). These results indicate that TAT-RP1 ameliorated radiation-induced skin injury in vivo.

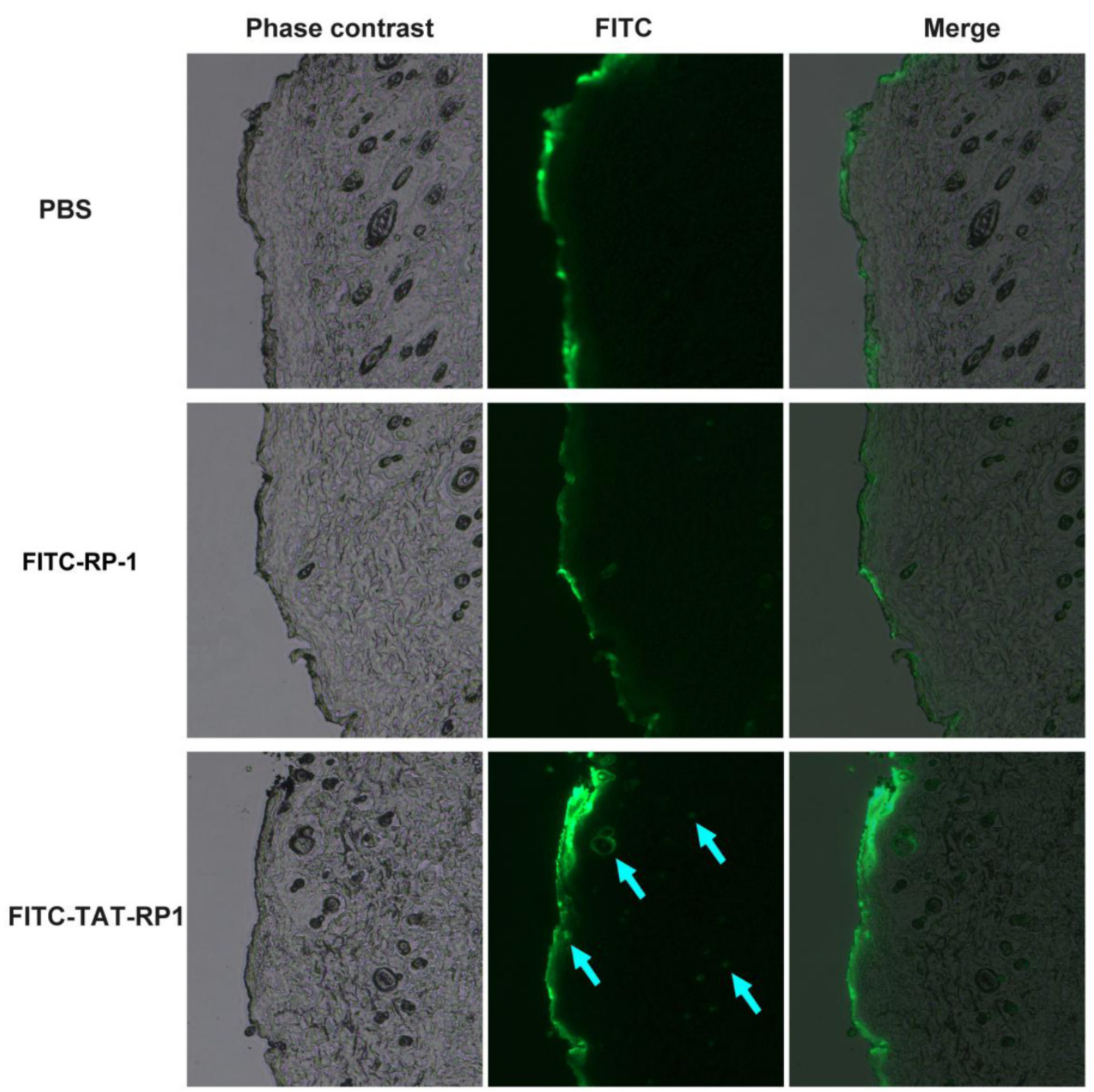

Figure 4. Penetration of TAT-RPI into rat skin. Fifty micrograms of indicated peptides were topically applied onto a shaved area of rat skin. Frozen sections of skin tissues were obtained $4 \mathrm{~h}$ after the application of peptides and observed by a fluorescent microscope. 

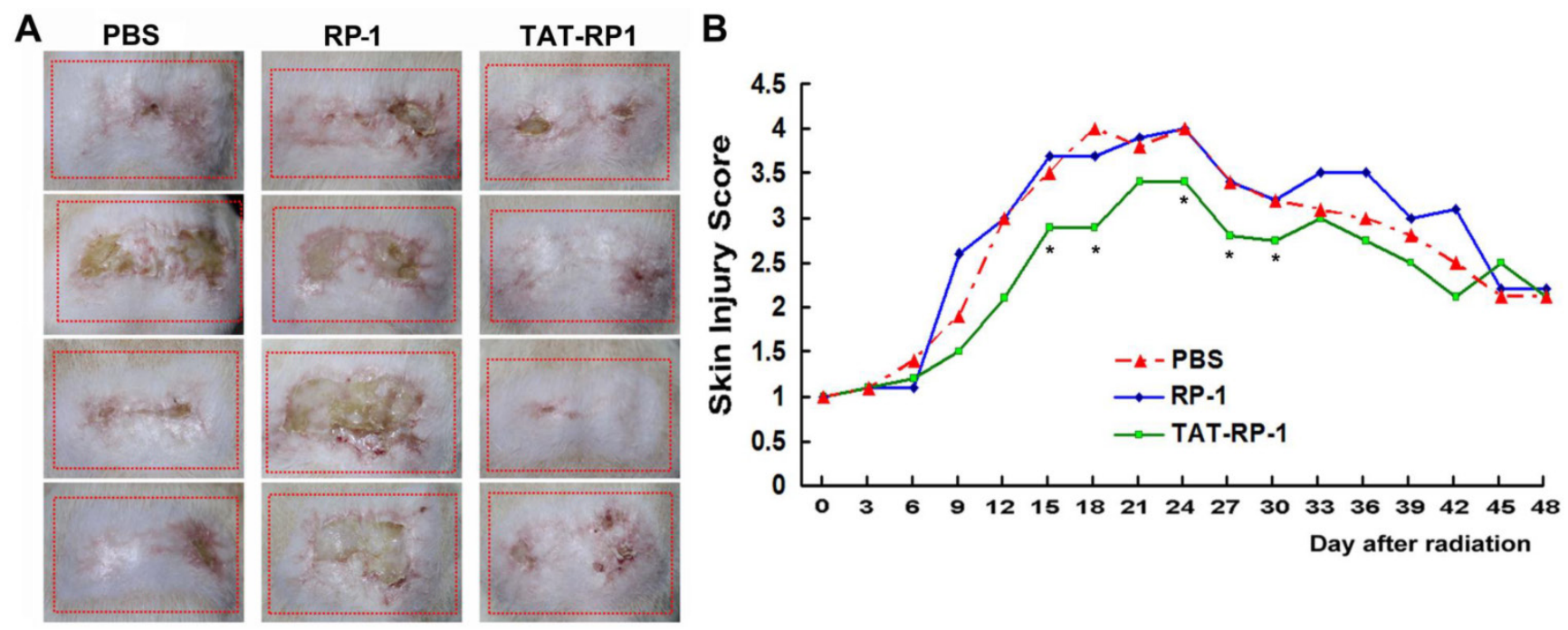

Figure 5. Effect of TAT-RPI on the amelioration of radiation-induced skin injury in vivo. (A) Representative skin images of different groups 30 days after irradiation. The dotted square represents irradiated skin area. (B) Effect of TAT-RPI on the amelioration of radiation-induced skin injury. Rats were irradiated to the buttock skin with a single dose of $45 \mathrm{~Gy}$ followed by a topical administration of indicated peptides ( 5 animals per group). Skin injury in these groups was measured using a semi-quantitative score of I (no damage) to 5 (severe damage). $* P<0.05$, compared with PBS-treated control group.

\section{Discussion}

During radiation exposure, skin damage occurs instantaneously, mediated by a burst of free radicals. Irradiated skin cells generate ROS, including oxygen ions, free radicals and peroxides. The detrimental ROS can further result in damage to nuclear DNA and alteration of proteins, lipids, and carbohydrates (23). Therefore, supplementation of antioxidant enzymes or compounds has been utilized to treat radiation-induced skin injury. Superoxide anion, one of the major detrimental ROS, can be converted to hydrogen peroxide by SODs. Virus-mediated SOD2 expression contributes to mitigation of radiation-induced skin injury in mice (24). Superoxide dismutase/catalase mimetic EUK-207 is able to reduce radiation dermatitis and promotion of wound healing using synthetic superoxide dismutase/catalase mimetic EUK-207 (25). We have previously reported that forced expression of antioxidant heme oxygenase-1 (HO-1) ameliorates radiation-induced skin injury in rats (21). In the present study, we synthesized TAT PTD coupled RP-1 peptide (TAT-RP1) and applied this new fusion peptide in the treatment of radiation-induced skin damage in vitro and in vivo. Results revealed that TAT-RP1 was efficiently incorporated into human $\mathrm{HaCaT}$ cells in a dose- and time-dependent manner. TAT-RP1 decreased HaCaT cell radiosensitivity to irradiation. Moreover, TAT-RP1 was able to penetrate the skin of rats and topical spread of TAT-RP1 ameliorated radiation-induced skin damage in rats.

PTD is strings of amino acids rich in arginine and lysine residues, which can convey molecules across the cell membrane barrier and improve the delivery of large molecules into cells. Since the discovery of HIV-TAT in 1988 (18,19), dozens of PTDs have been documented, including natural PTDs as well as synthetic ones (26-28). These PTDs can become powerful and promising tools as "protein therapy" (26-28). The classic HIV-TAT PTD does not enhance the immunogenicity when fused with peptides or proteins (29). Here, using the classical HIV-TAT PTD, we synthesized TAT-RP1 fusion peptide, which mediated the incorporation of antioxidant RP-1 into HaCaT cells, while the natural RP-1 peptide cannot penetrate cytomembrane barrier. The fusion of TAT PTD also decreased cell radiosensitivity to irradiation and increased total antioxidant capability of $\mathrm{HaCaT}$ cells. These results suggest that TAT-RP1 has potential as a protein therapy for radiation-induced skin injury.

The possible advantage for TAT-RP1 is that the PTD can mediate protein internalization into cells indiscriminately, and its required dose can be controlled exactly, decreasing potential side effects. Moreover, TAT-RP1 exhibited a protective role after 4 $\mathrm{h}$ of pretreatment. This result indicated that PTD-based delivery provided rapid therapy that was much faster than most gene transfer-based therapy. The protein transduction domain-based approach has provided a promising therapeutic alternative for radiation-induced injury.

In addition to its ability to cross the cell membrane, HIV-TAT can also mediate the penetration of skin tissue $(30,31)$. Here, we showed that four hours after topical administration of TAT-RP1, fluorescence could be detected in both the epidermis and skin eppendage of rat skin. Skin tissue consists of the stratum corneum, epidermis and dermis (32) and the obstacles 
of skin penetration lie in the stratum corneum, which blocks the penetration of extraneous molecules as a self-protection barrier $(33,34)$. The stratum corneum is composed of thick layers of collagen and glycoprotein. The mechanism of skin penetration is still under exploration; however, searching for a skin-penetrating PTD is meaningful because it directly contributes to the treatment for skin-related diseases. Administration of drugs through the skin surface is desirable for skin injury, while mainly hampered because of the limitation in the molecular weight of target protein. It is commonly accepted that proteins or large peptides, whose molecular weights are usually above $500 \mathrm{Da}$, can hardly be absorbed by skin and utilized as skin-based drugs $(34,35)$. However, the application of TAT PTD overcomes this limitation. Our results indicated that TAT can mediate the internalization of heterogeneous RP-1 peptide into skin tissues and attenuated radiation-induced skin injury in rats. We postulate that the TAT PTD is also able to facilitate other proteins through the stratum corneum barrier and TAT-RP1 may be applied for other vertebrates and other kind of skin injuries.

\section{Conclusions}

In summary, the synthesized and purified TAT-RP1 peptide was incorporated into human Ha$\mathrm{CaT}$ cells in a dose- and time-dependent manner without significant cytotoxicity. TAT-RP1 decreased radiosensitivity to irradiation in $\mathrm{HaCaT}$ cells. Moreover, TAT-RP1 was able to penetrate the skin of rats. Topical application of TAT-RP1 ameliorated radiation-induced skin damage in rats. These results suggest that TAT-RP1 has potential as a protein therapy for radiation-induced skin injury.

\section{Acknowledgements}

This work is supported by the National Natural Science Foundation of China (81102078, 81172597, 81372433 and 81270237), Natural Science Foundation of Jiangsu Province (BK2011165 and BK20131149) and the Priority Academic Program Development of Jiangsu Higher Education Institutions (PAPD).

\section{Competing Interests}

The authors have declared that no competing interest exists.

\section{References}

1. Thompson IM, Jr., Tangen CM, Paradelo J et al. Adjuvant radiotherapy for pathologically advanced prostate cancer: a randomized clinical trial. Jama 2006;296:2329-2335.

2. Sprung $\mathrm{CN}$, Chao $\mathrm{M}$, Leong $\mathrm{T}$ et al. Chromosomal radiosensitivity in two cell lineages derived from clinically radiosensitive cancer patients. Clin Cancer Res 2005;11:6352-6358.

3. Miller DL, Balter S, Noonan PT et al. Minimizing radiation-induced skin injury in interventional radiology procedures. Radiology 2002;225:329-336.
4. 0. Simonen P, Hamilton C, Ferguson $S$ et al. Do inflammatory processes contribute to radiation induced erythema observed in the skin of humans? Radiother Oncol 1998, 46:73-82.

5. Hymes SR, Strom EA, Fife C. Radiation dermatitis: clinical presentation, pathophysiology, and treatment 2006. J Am Acad Dermatol 2006, 54:28-46.

6. Ryan JL. Ionizing radiation: the good, the bad, and the ugly. J Invest Dermatol 2012;132:985-993.

7. Palmer DM, Kitchin JS. Oxidative damage, skin aging, antioxidants and a novel antioxidant rating system. J Drugs Dermatol 2010;9:11-15.

8. Shindo Y, Witt E, Packer L. Antioxidant defense mechanisms in murine epidermis and dermis and their responses to ultraviolet light. J Invest Dermatol 1993;100:260-265.

9. Chevion S, Berry EM, Kitrossky $\mathrm{N}$ et al. Evaluation of plasma low molecular weight antioxidant capacity by cyclic voltammetry. Free Radic Biol Med 1997;22:411-421.

10. Schallreuter KU, Wood JM: Free radical reduction in the human epidermis. Free Radic Biol Med 1989, 6(5):519-532.

11. Duda TF, Jr., Vanhoye D, Nicolas P. Roles of diversifying selection and coordinated evolution in the evolution of amphibian antimicrobial peptides. $\mathrm{Mol}$ Biol Evol 2002;19:858-864.

12. Clarke BT. The natural history of amphibian skin secretions, their normal functioning and potential medical applications. Biol Rev Camb Philos Soc 1997;72:365-379.

13. Liu C, Hong J, Yang $\mathrm{H}$ et al. Frog skins keep redox homeostasis by antioxidant peptides with rapid radical scavenging ability. Free Radic Biol Med 2010;48:1173-1181.

14. Yang $\mathrm{H}$, Wang $\mathrm{X}$, Liu $\mathrm{X}$ et al. Antioxidant peptidomics reveals novel skin antioxidant system. Mol Cell Proteomics 2009;8:571-583.

15. Schwarze SR, Dowdy SF. In vivo protein transduction: intracellular delivery of biologically active proteins, compounds and DNA. Trends Pharmacol Sci 2000;21:45-48.

16. Embury J, Klein D, Pileggi A et al. Proteins linked to a protein transduction domain efficiently transduce pancreatic islets. Diabetes 2001;50:1706-1713.

17. Schwarze SR, Ho A, Vocero-Akbani A et al. In vivo protein transduction: delivery of a biologically active protein into the mouse. Science 1999;285:1569-1572.

18. Green M, Loewenstein PM. Autonomous functional domains of chemically synthesized human immunodeficiency virus tat trans-activator protein. Cell 1988;55:1179-1188

19. Frankel AD, Pabo CO. Cellular uptake of the tat protein from human immunodeficiency virus. Cell 1988;55:1189-1193.

20. Joliot A, Prochiantz A. Transduction peptides: from technology to physiology. Nat Cell Biol 2004;6:189-196.

21. Zhang S, Song C, Zhou J et al. Amelioration of radiation-induced skin injury by adenovirus-mediated heme oxygenase- 1 (HO-1) overexpression in rats. Radiat Oncol 2012;7:4.

22. Kohl RR, Kolozsvary A, Brown SL et al. Differential radiation effect in tumor and normal tissue after treatment with ramipril, an angiotensin-converting enzyme inhibitor. Radiat Res 2007, 168:440-445.

23. Anand AJ, Dzik WH, Imam A et al. Radiation-induced red cell damage: role of reactive oxygen species. Transfusion 1997;37:160-165.

24. Yan S, Brown SL, Kolozsvary A et al. Mitigation of radiation-induced skin injury by AAV2-mediated MnSOD gene therapy. J Gene Med 2008;10:1012-1018.

25. Doctrow SR, Lopez A, Schock AM et al. A synthetic superoxide dismutase/catalase mimetic EUK-207 mitigates radiation dermatitis and promotes wound healing in irradiated rat skin. $J$ Invest Dermatol 2012;133:1088-1096.

26. Murriel CL, Dowdy SF. Influence of protein transduction domains on intracellular delivery of macromolecules. Expert opinion on drug delivery 2006, 3(6):739-746.

27. van den Berg A, Dowdy SF. Protein transduction domain delivery of therapeutic macromolecules. Current opinion in biotechnology 2011, 22(6):888-893.

28. Ho A, Schwarze SR, Mermelstein SJ et al. Synthetic protein transduction domains: enhanced transduction potential in vitro and in vivo. Cancer Res 2001;61:474-477.

29. Leifert JA, Harkins S, Whitton JL. Full-length proteins attached to the HIV tat protein transduction domain are neither transduced between cells, nor exhibit enhanced immunogenicity. Gene Ther 2002;9:1422-1428.

30. Jin LH, Bahn JH, Eum WS et al. Transduction of human catalase mediated by an HIV-1 TAT protein basic domain and arginine-rich peptides into mammalian cells. Free Radic Biol Med 2001;31:1509-1519.

31. Lopes LB, Brophy CM, Furnish E et al. Comparative study of the skin penetration of protein transduction domains and a conjugated peptide. Pharm Res 2005;22:750-757.

32. Gniadecka M, Faurskov Nielsen $\mathrm{O}$, Christensen $\mathrm{DH}$ et al. Structure of water, proteins, and lipids in intact human skin, hair, and nail. J Invest Dermatol 1998;110:393-398.

33. Taylor SC: Skin of color biology, structure, function, and implications for dermatologic disease. J Am Acad Dermatol 2002, 46:S41-62.

34. Bos JD, Meinardi MM. The 500 Dalton rule for the skin penetration of chemical compounds and drugs. Exp Dermatol 2000;9:165-169.

35. Norlen L. Skin barrier structure and function: the single gel phase model. J Invest Dermatol 2001;117:830-836. 